\title{
Default Mode Dynamics for Global Functional Integration
}

\author{
Deniz Vatansever, ${ }^{1,2}$-David K. Menon, ${ }^{1,2}$ Anne E. Manktelow, ${ }^{1,2}$ Barbara J. Sahakian, ${ }^{3}$ and Emmanuel A. Stamatakis ${ }^{1,2}$ \\ ${ }^{1}$ Division of Anaesthesia and ${ }^{2}$ Wolfson Brain Imaging Centre, Department of Clinical Neurosciences, and ${ }^{3}$ Department of Psychiatry, School of Clinical \\ Medicine, University of Cambridge, Cambridge CB2 0QQ, United Kingdom
}

The default mode network (DMN) has been traditionally assumed to hinder behavioral performance in externally focused, goal-directed paradigms and to provide no active contribution to human cognition. However, recent evidence suggests greater DMN activity in an array of tasks, especially those that involve self-referential and memory-based processing. Although data that robustly demonstrate a comprehensive functional role for DMN remains relatively scarce, the global workspace framework, which implicates the DMN in global information integration for conscious processing, can potentially provide an explanation for the broad range of higher-order paradigms that report DMN involvement. We used graph theoretical measures to assess the contribution of the DMN to global functional connectivity dynamics in 22 healthy volunteers during an fMRI-based $n$-back working-memory paradigm with parametric increases in difficulty. Our predominant finding is that brain modularity decreases with greater task demands, thus adapting a more global workspace configuration, in direct relation to increases in reaction times to correct responses. Flexible default mode regions dynamically switch community memberships and display significant changes in their nodal participation coefficient and strength, which may reflect the observed whole-brain changes in functional connectivity architecture. These findings have important implications for our understanding of healthy brain function, as they suggest a central role for the DMN in higher cognitive processing.

Key words: alluvial diagram; default mode network; flexibility; functional connectivity; graph theory; large-scale brain network

\section{Significance Statement}

The default mode network (DMN) has been shown to increase its activity during the absence of external stimulation, and hence was historically assumed to disengage during goal-directed tasks. Recent evidence, however, implicates the DMN in self-referential and memory-based processing. We provide robust evidence for this network's active contribution to working memory by revealing dynamic reconfiguration in its interactions with other networks and offer an explanation within the global workspace theoretical framework. These promising findings may help redefine our understanding of the exact DMN role in human cognition.

\section{Introduction}

Recent progress in MRI data acquisition and analysis has advanced our understanding that the human brain is organized into distinct, yet interacting large-scale brain networks (LSNs; Damoiseaux et al., 2006; De Luca et al., 2006). However, one robust

Received June 3, 2015; revised Aug. 17, 2015; accepted Sept. 13, 2015.

Author contributions: D.K.M., A.E.M., B.J.S., and E.A.S. designed research; D.K.M., A.E.M., B.J.S., and E.A.S. performed research; D.V., D.K.M., A.E.M., B.J.S., and E.A.S. contributed unpublished reagents/analytic tools; D.V., D.K.M., and E.A.S. analyzed data; D.V., D.K.M., A.E.M., B.J.S., and E.A.S. wrote the paper.

This work was supported by the Evelyn Trust (RUAG/018). In addition, D.V. received funding from the Yousef Jameel Academic Program; D.K.M. is supported by the National Institute for Health Research (NIHR) Cambridge Biomedical Centre (RCZB/004) and an NIHR Senior Investigator Award (RCZB/014); and E.A.S. is supported by the Stephen Erskine Fellowship, Queens' College Cambridge. We also thank Dr. Sanja Abbott for programming the stimulus delivery, and Dr. Guy Williams and Victoria Lupson and the rest of the staff in the Wolfson Brain Imaging Centre at Addenbrooke's Hospital for their assistance in scanning. Last, we thank all the participants for their contribution to this study.

The authors declare no competing financial interests.

This article is freely available online through the J Neurosci Author Open Choice option.

Correspondence should be addressed to Deniz Vatansever, Department of Clinical Neurosciences, Division of Anaesthesia, University of Cambridge, Box 93, Addenbrooke's Hospital, Hills Road, Cambridge CB2 0QQ, UK. E-mail: ddsv2@cam.ac.uk.
LSN comprising the posterior cingulate, medial prefrontal cortices, and angular gyri continues to puzzle the scientific community in regard to its cognitive significance (Buckner et al., 2008). Termed default mode network (DMN; Raichle et al., 2001), this set of regions has been reported to decrease its activity during attention-demanding paradigms (Shulman et al., 1997; Mazoyer et al., 2001), and it has been assumed to interfere with task performance (Spreng, 2012).

Challenging this notion of the $\mathrm{DMN}^{\prime}$ s cognitive irrelevance, emerging studies report greater DMN activity/connectivity in a range of tasks that require self-referential processing, such as autobiographical memory retrieval and future planning, as well as in social cognitive paradigms of empathizing, moral judgment, and narrative comprehension (Buckner et al., 2008; Spreng and Grady, 2010; Andrews-Hanna, 2012). Additionally, there is evi-

This is an Open Access article distributed under the terms of the Creative Commons Attribution License Creative Commons Attribution 4.0 International, which permits unrestricted use, distribution and reproduction in any medium provided that the original work is properly attributed. 
dence suggesting (1) changes in the $\mathrm{DMN}^{\prime}$ s spatial extent during task execution (Spreng et al., 2013; Vatansever et al., 2015), (2) positive correlations between DMN connectivity and behavioral measures (Hampson et al., 2006), and (3) DMN interactions with other LSNs during rest (Fox et al., 2005) and task conditions (Spreng et al., 2010). Overall, these findings point to a fundamental cognitive function for the DMN that is yet to be precisely delineated.

Given such involvement in a wide range of tasks, extensive communication with other networks, and its central placement in the brain from the perspectives of both anatomical and functional connectivity (Hagmann et al., 2008; Buckner et al., 2009; van den Heuvel and Sporns, 2013), the DMN may play a role in the global integration of information (van den Heuvel and Sporns, 2011; Braga et al., 2013) necessary for conscious processing during both unconstrained rest and controlled task conditions. This concept overlaps with the theoretical account of a global workspace originally proposed by Baars (Baars, 2002) and may mechanistically involve the DMN and dorsal attention network competing for limited resources facilitated by the frontoparietal network through long-range, flexible connections (Dehaene and Changeux, 2011; Smallwood et al., 2012). As a hub of this global workspace, the DMN may generate the necessary associative information to be retained and manipulated by the frontoparietal network.

From a network organization perspective, the brain is considered to be economically configured into a cost-effective, highly modular, small-world architecture that flexibly adapts a more expensive, yet informatically efficient and integrated global workspace in response to environmental demands (Bullmore and Sporns, 2012). Given our hypothesis about the potential contribution of DMN to the global integration of information, we investigated in this study the alterations in whole-brain interactions in relation to performance during an $n$-back working-memory task with parametric increase in difficulty, specifically focusing on the DMN's involvement in wholebrain reconfiguration.

For the purpose of quantifying LSN interactions, we focused on modularity, a graph theoretical metric used to calculate the level of integration and segregation across brain regions in a given system (Newman, 2006; Meunier et al., 2009b), as well as global variable connectivity (Cole et al., 2013), nodal participation coefficient, and nodal strength (Rubinov and Sporns, 2011), which describe the regional contribution of network nodes to global changes in functional connectivity.

Given the association between effortful task performance and modular brain organization (van den Heuvel et al., 2009), we hypothesized that modularity would decrease with increasing cognitive effort. Additionally, based on existing literature on the engagement of DMN regions in a diverse set of goal-directed paradigms and their multisynaptic characteristics with extensive structural and functional connections to the rest of the brain, we predicted that the decrease in modularity and the expansion of global workspace topology would be reflected by the changes in DMNs' interactions with other LSNs, supporting a potential role for $\mathrm{DMN}$ as a global integrator of information.

\section{Materials and Methods}

Participants. After the study proposal was approved by the local ethics committee, informed consent was obtained from 22 right-handed healthy participants (age range, $19-57$ years; mean age, 35.0 years; $S D=$ 11.2; female-to-male ratio, 9/13), all of whom took part in the $n$-back working-memory experiment as well as four other cognitive paradigms that are not reported in this study. The average score for the measure of premorbid IQ via the National Adult Reading Test was 117.1 (SD = 5.76). Meanwhile, results of the Mini Mental State Exam averaged 29.33 $(\mathrm{SD}=0.85)$. Thus, no signs of memory problems were detected. In addition, no history of drug or alcohol abuse, psychiatric or neurological disorders, or head injury was recorded in any of the participants.

Image acquisition. The experiment was conducted in a Siemens Trio 3T scanner at the Wolfson Brain Imaging Centre, Addenbrooke's Hospital, Cambridge. The imaging session began with a localizer, followed by a high-resolution, T1-weighted, magnetization-prepared, $180^{\circ}$ radiofrequency pulses and rapid gradient-echo structural scan $[\mathrm{TR}=2300 \mathrm{~ms}$; $\mathrm{TE}=2.98 \mathrm{~ms} ; \mathrm{TA}=9.14 \mathrm{~min}$; flip angle, $9^{\circ}$; field-of-view $(\mathrm{FOV}) \mathrm{read}$, $256 \mathrm{~mm}$; voxel size, $1.0 \times 1.0 \times 1.0 \mathrm{~mm}$; slices per slab, 176]. Wholebrain echo planar imaging was used for the $n$-back paradigm $(\mathrm{TR}=2000$ $\mathrm{ms}$; TE = $30 \mathrm{~ms}$; flip angle, $78^{\circ}$; FOV read, $192 \mathrm{~mm}$; voxel size, $3.0 \times$ $3.0 \times 3.0 \mathrm{~mm}$; volumes, 345 ; slices per volume, 32 ).

Paradigm specifications. In the $n$-back working-memory paradigm, three fixation blocks were pseudorandomly interleaved with five cycles of four $n$-back blocks ranging in difficulty between 0 -back and 3 -back. Single letters in white font were presented serially on a black background for $500 \mathrm{~ms}$, each followed by $2500 \mathrm{~ms}$ fixation on a cross. While in the 0 -back trials, participants were requested to press a button with their left index finger on the appearance of the letter $\mathrm{Z}$ in a string of random letters. More difficult levels of $n$-back required the same button press in response to a match between the current and one previous letter (1-back), two previous letters (2-back), or three previous letters (3-back). The participants also responded to nontargets by pressing a button under their right-hand middle finger. Each trial, including the fixation and task blocks, lasted $36 \mathrm{~s}$, and 10-s-long instructions were presented before each block.

Spatial and temporal preprocessing. The preprocessing and image analysis were performed using Statistical Parametric Mapping (SPM) Version 8.0 (http://www.fil.ion.ucl.ac.uk/spm/) and Matlab Version 12a platforms (http://www.mathworks.co.uk/products/matlab/). The first six volumes were removed to eliminate saturation effects and achieve steady-state magnetization. The remaining data were slice-time adjusted, motion corrected, normalized to the Montreal Neurological Institute (MNI) space by using the segmented high-resolution gray matter structural image and a gray matter template. The final preprocessing step involved smoothing the images with an $8 \mathrm{~mm}$ FWHM Gaussian kernel. The resulting data were used for statistical modeling.

A strict temporal preprocessing pipeline of nuisance regression included motion and CompCor components attributable to the signal from white matter and CSF (Behzadi et al., 2007), as well as a linear detrending term, eliminating the need for global signal normalization (Murphy et al., 2009; Chai et al., 2012). The subject-specific six realignment parameters, the main effect of task condition, and their first order derivatives were also included in the analysis as potential confounds (Fair et al., 2007). Moreover, a temporal filter of 0.009 and $0.08 \mathrm{~Hz}$ was applied to focus on low-frequency fluctuations (Fox et al., 2005).

Functional connectivity and graph theoretical analyses. The main objectives of our study were to examine the whole-brain connectivity changes in response to increasing task difficulty and to assess the alterations in the interaction of DMN regions with other LSNs. Thus, we initially used a whole-brain approach, in which average correlation matrices based on 264 ROIs (Power et al., 2011), corresponding to 10 well established LSNs, formed the basis of our functional connectivity and subsequent modularity analyses. The results, visualized via circular and novel alluvial representations (Rosvall et al., 2009), aimed to explicate the modular organization of the brain across task difficulty, but also were intended to clarify the change in communities formed by the LSNs and possible behavioral correlations. While the flexibility of the 264 nodes was assessed using the global variable connectivity measure, the DMN regions' nodal participation coefficient and strength were further scrutinized for a full characterization of DMNs' contribution to the global connectivity dynamics.

Definition of ROIs. We adopted a set of 264 brain regions based on both resting (Cohen et al., 2008) and task (Power et al., 2011) functional connectivity meta-analyses that have been shown to produce reliable network topologies (Dosenbach et al., 2007; Power et al., 2011; Cole et al., 
2013; Spreng et al., 2013). As opposed to voxelwise or anatomical definitions, the selected set of ROIs minimize signal overlap from multiple functional regions (Wig et al., 2011). The network partitions outlined by Cole et al. (2013) were used to assign each one of the 264 ROIs to one of the 14 LSNs documented in the original publication (Power et al., 2011). These 14 network partitions included 10 well established networks: the dorsal attention network, the ventral attention network, the salience network, the cingulo-opercular network, the frontoparietal network, the DMN, the visual network, the auditory network, the somatomotor network (hand and mouth), and the subcortical network. The partitions also included three networks within the memory retrieval network, the cerebellum network, and a network of uncertain function. As in the original publication, the uncertain nodes were not related to any of the known LSNs (Power et al., 2011).

Correlation matrices. We used the Conn functional connectivity toolbox (Whitfield-Gabrieli and Nieto-Castanon, 2012) to construct task-specific (Fixation, 0-back, 1-back, 2-back, 3-back) functional connectivity matrices. For this purpose, the BOLD time series were first divided into block-specific scans as indicated by the onsets and durations of each task block. The delay in hemodynamic response was accounted for by convolving the block regressors for each task condition with a rectified hemodynamic response function. For each task condition, the scans associated with nonzero effects in the resulting time series were concatenated and weighted by the value of the corresponding time series. This procedure not only adds the expected hemodynamic delay to different task blocks, but also de-weights the initial and final scans within each task block when computing functional correlation measures to avoid spurious jumps in BOLD signal at the points of concatenation and to minimize the potential cross talk between adjacent task blocks (Whitfield-Gabrieli and Nieto-Castanon, 2012).

Following this concatenation procedure, undirected and weighted matrices $(264 \times 264)$ of Fisher $z$-transformed bivariate correlation coefficients (Pearson's $r$ ) were constructed for each experimental condition (Fixation, 0-Back, 1-Back, 2-Back, and 3-Back) and each subject using the average signal from the $6 \mathrm{~mm}$ spheres placed on the MNI coordinates for all 264 ROIs described above. The matrices reflected both positive and negative correlations. The arbitrary thresholding and binarization processes in graph theoretical analysis often lead to loss of information, especially in the case of negative correlations (Rubinov and Sporns, 2011), which is why we focused on the fully connected weightedcorrelation matrices.

Modularity analysis and behavioral correlation. Following the ROI selection and matrix construction steps, the correlation matrices with 264 ROIs as nodes and the weighted correlation coefficients as edges were first converted from Matlab to Pajek (Program for Large Network Analysis) format (Nooy et al., 2011). For the whole-brain, group-level modularity analysis, the resulting matrices were averaged across subjects. The aim was to quantify the partitioning of a functional network into communities of dense intramodule and sparse intermodule connections (Rubinov and Sporns, 2010). For each condition, including Fixation and the four levels of difficulty, the average correlation matrices were significance-clustered into modules using an Infomax community detection algorithm over 1000 bootstrap resampling and 10 partitioning iterations at the 0.05 level of significance (Rosvall and Bergstrom, 2010).

To make a statistical inference on the change in modularity with increasing task difficulty, the 0-Back control (low demand) and 3-Back task conditions (high demand) were chosen for comparison. The Louvain modularity Q score based on the Brain Connectivity Toolbox (Rubinov and Sporns, 2010) was calculated on weighted correlation matrices (Blondel et al., 2008; Rubinov and Sporns, 2011) for each subject at 0 -Back and 3-Back conditions, over 10 iterations. The highest $\mathrm{Q}$ with the greatest partitioning score was selected as the representative modularity score (Stanley et al., 2014). Using the GraphVar toolbox (Kruschwitz et al., 2015), a group-varying paired $t$ test was performed over 10 iterations to test the change in modularity at the 0.05 level of significance.

Linear regression analysis between 0-back Q scores and the change in Q scores between 0-back and 3-back highlighted the individual differences (corrected for age) in the relationship between baseline modularity and the potential change with increasing task difficulty. Given previous studies on the effect of age on structural connectivity (Stamatakis et al., 2011), functional connectivity, modularity (Meunier et al., 2009a), and cognitive task performance (Li and Sikström, 2002; Meunier et al., 2014), age was introduced as a potential confound for the linear regressions to account for the wide age range in our sample.

For a behavioral analysis, the reaction times to correct responses were first averaged across all trials and all blocks for each subject, separately for each level of task difficulty (0-back, 1-back, 2-back, and 3-back). The data were assessed for normality using the Shapiro-Wilk test and Q-Q plots. One outlier was removed as identified by the outlier-labeling rule (Hoaglin et al., 1986). Using a linear regression analysis, we correlated the change in modularity with the change in reaction time to correct responses between 0 -Back and 3-Back conditions to assess the behavioral significance of modularity (corrected for age). Although the reaction times to correct responses were chosen to represent task performance, in line with current literature (Kitzbichler et al., 2011), we have also calculated the $\mathrm{d}^{\prime}$ metric based on the signal detection theory for performance accuracy (Green and Swets, 1974) and performed paired $t$ tests to assess the expected decrease in $\mathrm{d}^{\prime}$ and increase in reaction time to correct responses between 0 -back and 3-back, and to confirm greater task difficulty with increasing $n$-back levels.

Nodal flexibility, participation coefficient, and strength. Having investigated the changes in modularity and the possible behavioral correlations across 22 subjects, our next objective was to clearly visualize the changes in community memberships responsible for the reconfiguration of the global brain modular architecture. The calculated communities were represented here using an alluvial diagram (Rosvall et al., 2009), which clearly outlines the interaction between LSNs at different difficulty levels, thus highlighting the flexible nodes that change community memberships. The 264 ROIs partitioning into 10 well established networks was color coded to aid the visualization of changes in community membership across the five distinct experimental conditions.

In addition, a novel graph theoretical metric called global variable connectivity (GVC) was used to assess each node's flexibility score across the five experimental conditions (Cole et al., 2013). GVC, calculated as the SD of a given node's connectivity strength, indicates the node's tendency to shift functional connections with other nodes across multiple contexts. To further characterize the alterations in the DMN regions' contribution to the reconfiguration of global functional connectivity, we calculated the participation coefficient and nodal strength for positive and negative weights and compared them with paired $t$ tests between 0 -Back (low demand) and 3-Back (high demand) conditions, controlling for multiple comparisons using Bonferroni's correction. While the participation coefficient assesses the diversity of intermodular links established by a given node, the nodal strength metric calculates the sum of weights and number of positive/negative connections.

\section{Results}

\section{Global brain modularity decreases with increasing cognitive load}

The connectivity matrices of bivariate correlation coefficients (Pearson) clearly illustrated the 10 well established LSNs with strong intranetwork connectivity profiles (Fig. 1). However, correlation matrices alone do not quantify the dynamic changes in internetwork interactions with increasing task difficulty. When assessing such architectural reconfiguration of brain dynamics, modularity has been a metric of choice to characterize network connections that transiently change their configurations in response to task demands (Bassett et al., 2006). Using this metric, we found that the modularity of global brain connectivity decreases with increasing cognitive load, in line with results from an MEG study (Kitzbichler et al., 2011). At Fixation, 0-Back, and 1 -Back conditions, the whole-brain connectivity profile revealed four stand-alone communities. This number decreased down to three major communities at the 2-Back condition and to two communities at the 3-Back condition (Fig. 1). Paired $t$ tests between the 0-Back (low demand) and 3-Back (high demand) con- 

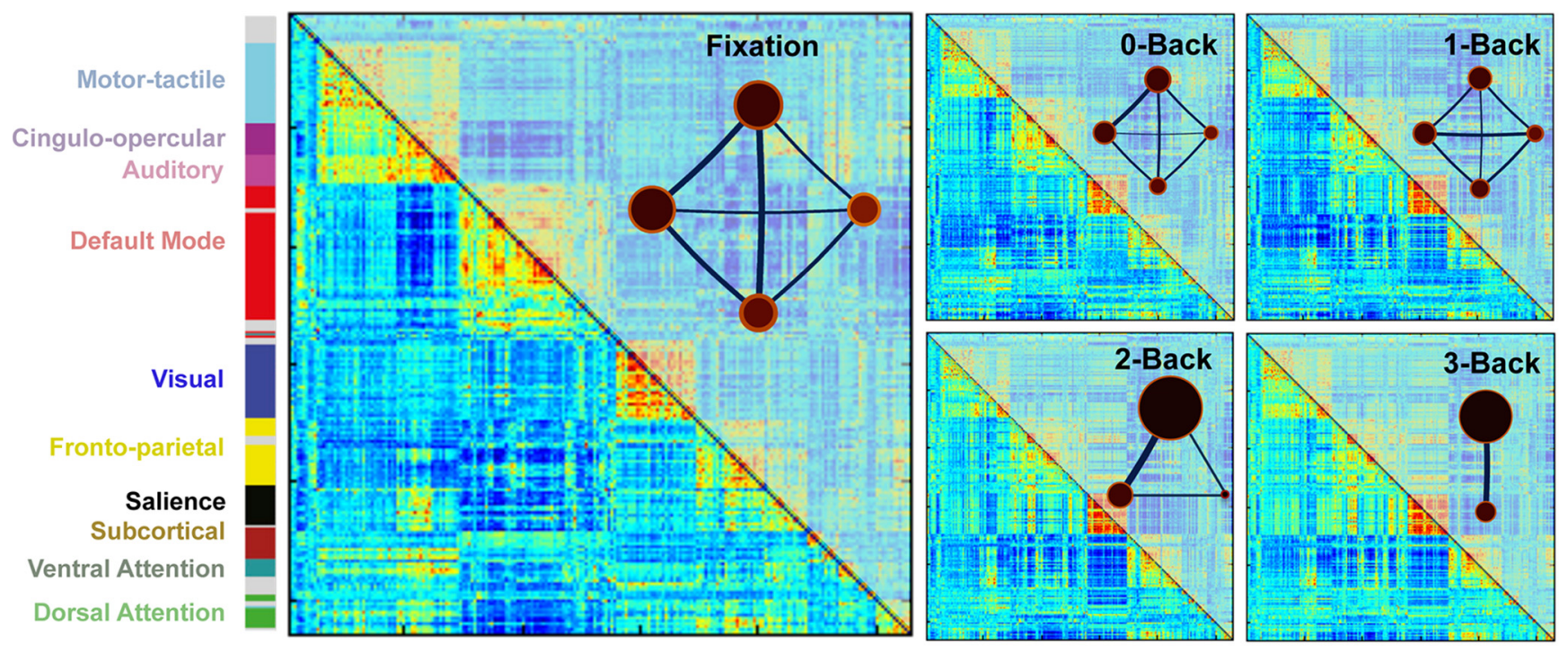

Figure 1. Global brain modularity decreases with increasing task demands. The correlation matrices denote bivariate (Pearson) correlation coefficients (red-blue scale, $\max =1.0, \min =-0.5$ ) for the five distinct experimental conditions of Fixation, 0-Back, 1-Back, 2-Back, and 3-Back, averaged across all subjects. The boxes with strong intranetwork correlations correspond to 10 well established LSNs from the existing literature (Cole et al., 2013). For modularity analysis, the Fisher-transformed Z values were significance clustered $(p<0.05)$ over 1000 bootstrap resampling and 10 partitioning iterations. The resulting modules are displayed using the circular visualization on the right-hand corner of the correlation matrices. The circle size and the line thickness of the links between the modules are representative of the average weights of the nodal connections.

A

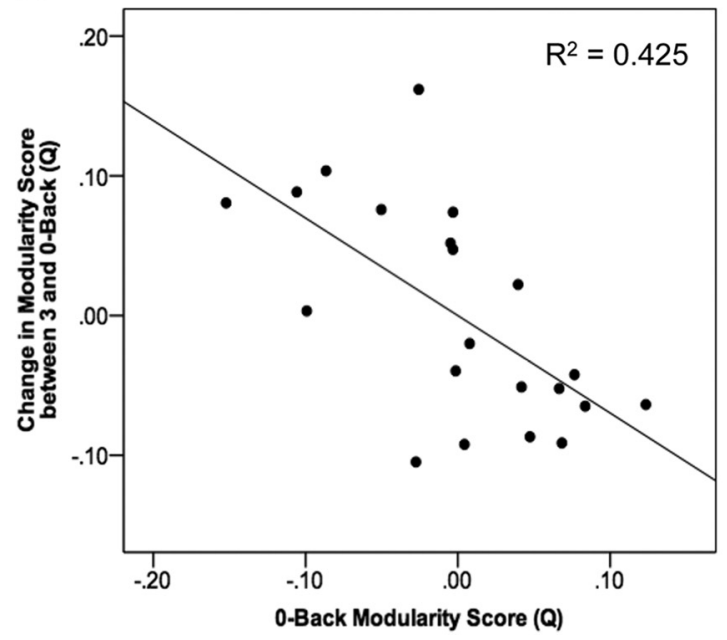

B

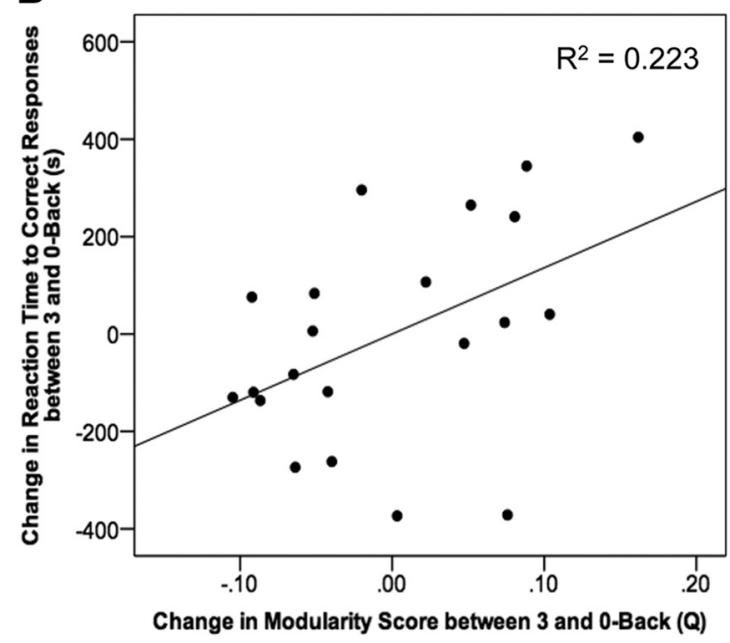

Figure 2. Individual differences in the change in modularity and their corresponding behavioral correlation. $A$, Participants with higher modularity $Q$ score at 0 -Back control condition demonstrated a smaller change in their modularity between 3-Back and 0-Back conditions $\left(r=-0.631, R^{2}=0.425, p=0.003\right)$. B. The change (3-Back minus 0 -Back) in subject-level $Q$ scores positively correlated with the change in the reaction time to correct responses between the two selected high-demand and low-demand $n$-back conditions $\left(r=0.469, R^{2}=0.223, p=0.037\right)$. Both linear regressions were corrected for age. Using the outlier identification technique, data from one volunteer were removed, as it was higher than the upper limit of the reaction time distribution. However, the same analyses with the outlier did not change the significance of the results $\left(\boldsymbol{A}: r=-0.617, R^{2}=0.405, p=0.003 ; \boldsymbol{B}: r=0.558, R^{2}=0.313, p=0.009\right)$.

ditions, over 10 randomized groups, suggested a significant decrease in modularity with increasing task load $(p=0.013)$. This outcome alludes to greater long-range interaction between LSNs and changes in brain topography toward a global workspace configuration (Baars, 2002) at the 3-Back condition. In other words, the brain adopts a more efficient, yet more costly organization in response to increasing cognitive demands (Kitzbichler et al., 2011).

\section{Change in modularity correlates with reaction time to correct responses}

Given the observed decrease in group-level modularity, our next objective was to investigate the individual differences in modu- larity changes and their potential correlation with behavioral scores obtained during task execution. For this purpose we first correlated the Louvain modularity Q score at 0 -Back condition with the change in $\mathrm{Q}$ score between 3-Back and 0-Back conditions, correcting for age. The results indicated a negative relationship, suggesting that the participants with higher modularity at the 0 -Back control condition showed a smaller change in their modularity when presented with the high-demand 3-Back condition, and vice versa $\left(r=-0.631, R^{2}=0.425, p=0.003\right.$; Fig. 2A).

Next we attempted to establish a relationship between modularity and behavior. At first, paired $t$ tests revealed a decrease in $\mathrm{d}^{\prime}$ $(p=9.40 \mathrm{E}-8)$ and an increase in reaction times to correct 


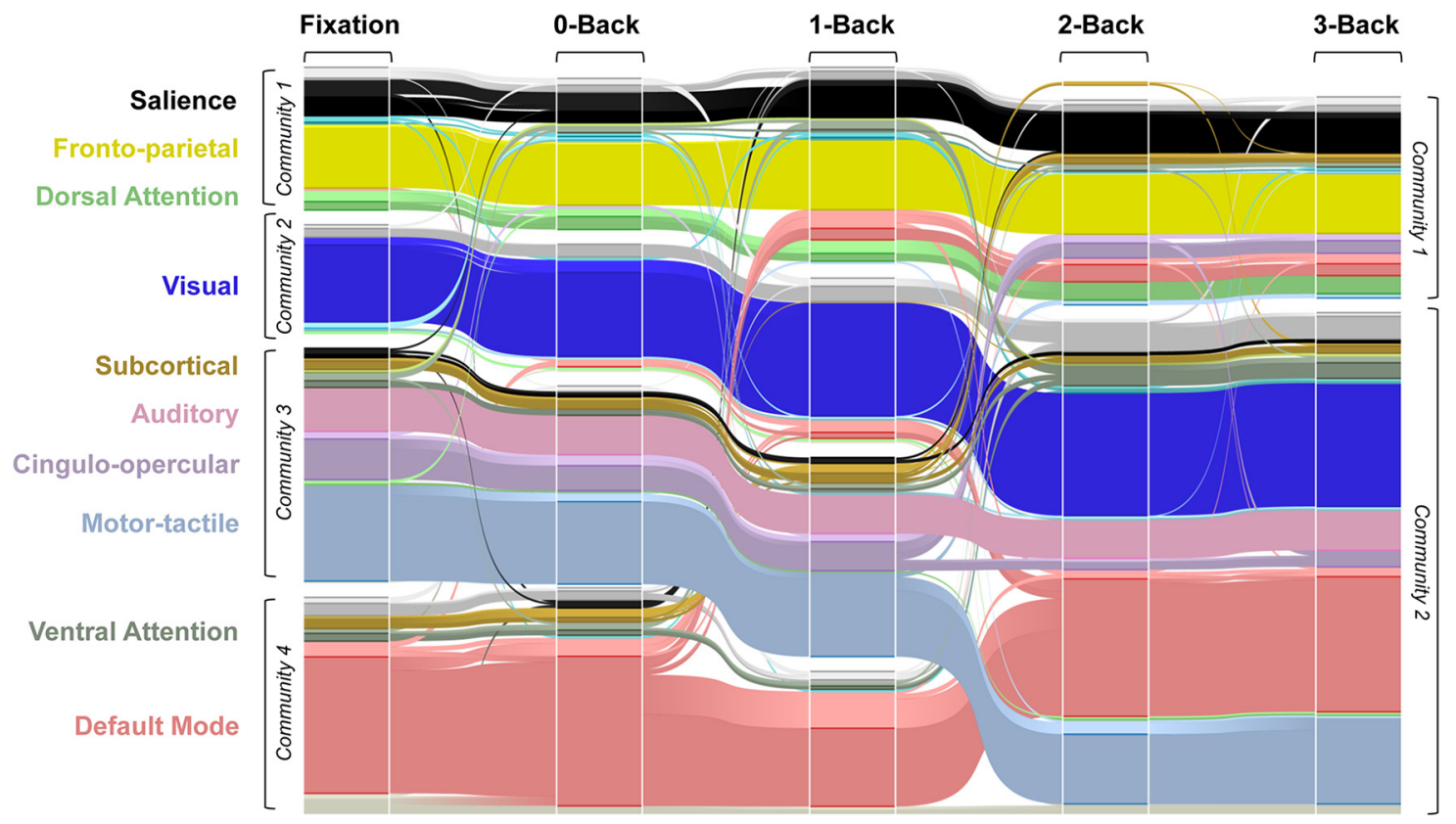

Figure 3. Dynamic changes in global brain connectivity across increasing task difficulty, represented by an alluvial diagram (Rosvall et al., 2009). At each task condition, the communities corresponding to the modules in Figure 1 are separated by white gaps. The 264 ROIs are color-coded based on their LSN memberships. The flows indicate the ROls, which switch community membership based on their functional similarity with other ROls in a given difficulty level. The darker shades in each network color denote the nodes that are part of a given module in $\geq 95 \%$ of the 1000 bootstrap partitioning iterations.

responses $(p=5.10 \mathrm{E}-5)$ when comparing 0-Back (mean: $\mathrm{d}^{\prime}=$ 3.45; reaction time, $619.26 \mathrm{~ms}$ ) with 3 -Back conditions (mean: $\mathrm{d}^{\prime}=2.19$; reaction time, $958.12 \mathrm{~ms}$ ), confirming greater task difficulty at higher levels of $n$-back. Subsequently, the change in modularity Q scores were correlated with the change in the reaction time to correct responses between 3-Back and 0-Back conditions for each subject, corrected for age. The results suggested that the subjects who displayed a higher change in modularity also showed a higher change in their reaction time $(r=0.469$, $R^{2}=0.223, p=0.037$; Fig. $2 B$ ), indicating a behavioral significance of the observed alterations in brain architecture. In other words, slower response in the high-demand 3-Back versus lowdemand 0 -Back condition was associated with greater brain modularity. Such results imply that worse performance may be linked to limited long-range integration among distant brain regions, thus a smaller global workspace configuration. Similar correlations with behavior and modularity were previously reported using the $\mathrm{d}^{\prime}$ metric between 1-Back and 2-Back conditions (Stanley et al., 2014).

\section{Global brain dynamics reveal flexible default mode regions}

Subsequent to the observed decrease in modularity with increasing cognitive load and the corresponding correlation with performance in the scanner, our aim was to scrutinize the exact changes in the global brain connectivity profile and the interaction of the DMN with other LSNs. Our hypothesis was that the DMN, in a global integrator role contributing to the global workspace, would show distributed interactions with a number of LSNs, reflected by the changes in community memberships with increasing task demands. The alluvial representation (Rosvall et al., 2009) provides a unique and informative tool for that purpose. The resulting diagram of whole-brain interactions indicated dynamic realignments in a number of default mode regions, revealing flexible nodes that switch memberships from one community to another, depending on cognitive demands.
Using the average, group-level modularity analysis for community detection discussed above, in the Fixation condition, Community 1 mainly comprised the salience, frontoparietal, and dorsal attention networks; Community 2, the visual network; Community 3, the subcortical, somatomotor, auditory, and cingulo-opercular networks; and Community 4, the ventral attention and default mode networks, respectively (Fig. 3). All 58 default mode regions were part of Community 4 except for a middle temporal gyrus node, which was more functionally similar to Community 1 . In addition to the DMN regions, Community 4 also included all the "memory retrieval" nodes, 46\% (13 of 28) of the uncertain nodes, and one salience node, namely the dorsal anterior cingulate cortex. Around $62 \%$ (8 of 13) of the subcortical nodes, which included the bilateral thalamic, but no striatal regions, also showed functional similarity with Community 4 .

However, this partitioning displayed transience with increasing task difficulty. In the 0-Back condition, the four modules remained stable relative to the Fixation condition with a number of salience network ROIs showing greater functional similarity with the DMN. The 1-Back condition displayed the greatest volatility in community membership, in which a portion of DMN regions from Community 4 switched to Community 1 and 2, encompassing the salience, frontoparietal, dorsal attention, and visual networks. In the 2-Back condition, the cingulo-opercular network ROIs were divided between two communities dominated by the frontoparietal and default mode networks, while some subcortical regions formed a separate community. At the 3-Back condition with the highest cognitive load, 17\% (10 of 58) of initial DMN regions changed their membership to Community 1 , whereas the remaining $48 \mathrm{DMN}$ regions have retained their community membership and formed an extensive Community 2 that included a number of somatosensory, cinguloopercular, auditory, visual, and subcortical regions.

This qualitative investigation was also supported by the GVC score, which assesses the flexibility of network nodes across task 


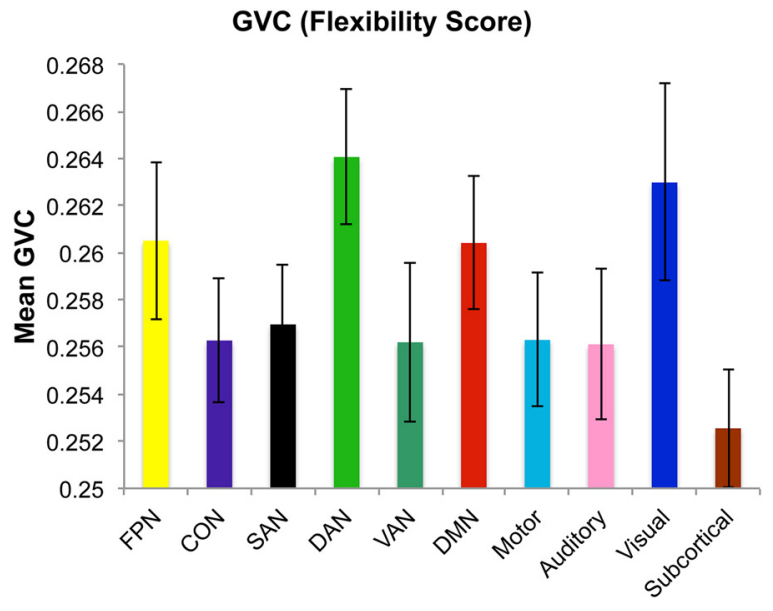

Figure 4. Mean global variable connectivity (GVC) score for the 10 LSNs across the five experimental conditions. GVC measures a given node's tendency to switch community memberships across different contexts (Cole et al., 2013). The color-coded bars illustrate the 10 well established LSNs' mean GVC, and the error bars show SE. The results indicate high flexibility in the DMN nodes (above the median score of 0.257), as well as in the frontoparietal, dorsal attention, and visual network nodes. FPN, Frontoparietal network; CON, cingulo-opercular network; SAN, salience network; DAN, dorsal attention network; VAN, ventral attention network.

conditions and was previously used in a study with 64 task states designating the frontoparietal and default mode as highly volatile networks (Cole et al., 2013). Across the five experimental conditions, the DMN regions showed high flexibility (above the median score of 0.257 ), a characteristic shared with the frontoparietal, dorsal attention, and visual network nodes (Fig. 4), which are commonly implicated in working-memory tasks with visual stimuli (Owen et al., 2005).

\section{Diversity of default mode connections decrease with increasing positive strength}

Having established that the modularity of the brain decreases with greater task load and that the DMN regions exhibit flexibility/volatility in community memberships, the subsequent aim of our study was to characterize the changes in DMN functional connectivity with greater task difficulty and to assess its contribution to global functional integration with further graph theoretical measures. For that purpose, we calculated the nodal participation coefficient and strength measures, which indicate the diversity of intermodular links and the number of positive/ negative connections of each node, respectively. From 0 -Back to 3-Back conditions, the DMN ROIs showed a significant decrease in their participation coefficient for both positive $(p=0.0006)$ and negative ( $p=3.53 \mathrm{E}-10)$ weights (Fig. $5 A$ ). However, the nodal strength increased for positive $(p=0.045)$ and decreased for negative ( $p=1.95 \mathrm{E}-10)$ weights, displaying a differential change in bidirectional functional connectivity to the rest of the brain (Fig. 5B).

Nodes with a high participation coefficient are believed to facilitate global integration between modules of a system (Guimerà and Amaral, 2005). In this case the significant decrease in the participation coefficient reflects the decrease of global brain modularity for both positive and negative weights. On the other hand, the increase in positive nodal strength alludes to a greater number of positive connections made with DMN regions, with a decrease in negative connections. Although the cognitive significance of anticorrelations is still speculative, recent evidence suggests biological relevance (Fox et al., 2009) and potential be- havioral significance (Kelly et al., 2008; Sala-Llonch et al., 2012); however, further empirical evidence is needed.

\section{Discussion}

Previous studies that aimed to describe the DMNs' contribution to cognitive processing have reported greater DMN involvement in a range of tasks assessing autobiographical memory retrieval, theory of mind, social cognition, episodic recall, and imagined scenes (Buckner et al., 2008; Andrews-Hanna et al., 2014). Important to consider in parallel are DMN activity/connectivity alterations observed in many neuropsychiatric conditions (Garrity et al., 2007; Whitfield-Gabrieli et al., 2009), traumatic brain injury (Sharp et al., 2011), normal aging (Damoiseaux et al., 2008), and under anesthesia (Stamatakis et al., 2010). Such evidence points toward a fundamental DMN function and necessitates a theoretical framework that can provide a comprehensive explanation for DMN involvement in many different forms of cognition and related disorders.

The aim of this study was to assess global brain connectivity changes with increasing cognitive demands in a working memory task and to determine a potential DMN involvement as a global integrator of information. Specifically, we used graph theoretical measures of modularity, global variable connectivity, and nodal participation coefficient and strength to assess the changing community architecture of the brain across increasing task difficulty in an $n$-back paradigm. The results showed that brain modularity decreased at higher levels of task load and this change was related to reaction time, indicating that the functional community formation is transient and that it changes in response to cognitive demands. Default mode ROIs displayed high flexibility and volatility in changing community memberships, with decreasing participation coefficient and increasing positive connectivity strength, thereby actively contributing to greater functional integration.

Such results highlight a fine balance between network segregation and integration in meeting task demands. Our findings are not only in line with reports demonstrating functional parcellation of the brain into densely intraconnected LSNs (Power et al., 2011), but also with studies that reveal dynamic internetwork interactions (de Pasquale et al., 2012; Spreng et al., 2013). In fact, a variety of neuroimaging techniques have proposed the economical organization of the brain into a small-world architecture that minimizes the cost of wiring and metabolism by forming and maintaining communities with a high number of local connections and few distant connections (Achard et al., 2006; Bullmore and Sporns, 2009, 2012). In this context, the DMN regions have been shown to represent rich clubs, i.e., areas of high global connectivity (van den Heuvel and Sporns, 2011; de Pasquale et al., 2013) that may serve as hubs for the integration of information. Similarly, the observed decrease in modularity with higher task load may be driven by changing DMN connectivity to the rest of the brain, demonstrated by the alluvial diagram as well as the significant changes in the diversity of intermodular links and the strength of connections made by DMN regions.

The highly stable modular architecture of the brain (Achard et al., 2006) has been previously reported to show transient network reconfiguration in response to changing environmental demands during simple tasks (Bassett et al., 2006). Moreover, modularity of the brain at rest was shown to predict subsequent performance in an $n$-back task (Stevens et al., 2012) and nodal flexibility was predictive of complex motor learning (Bassett et al., 2011), thus linking functional brain organization, learning, and memory. 
A Nodal Participation Coefficient (P)
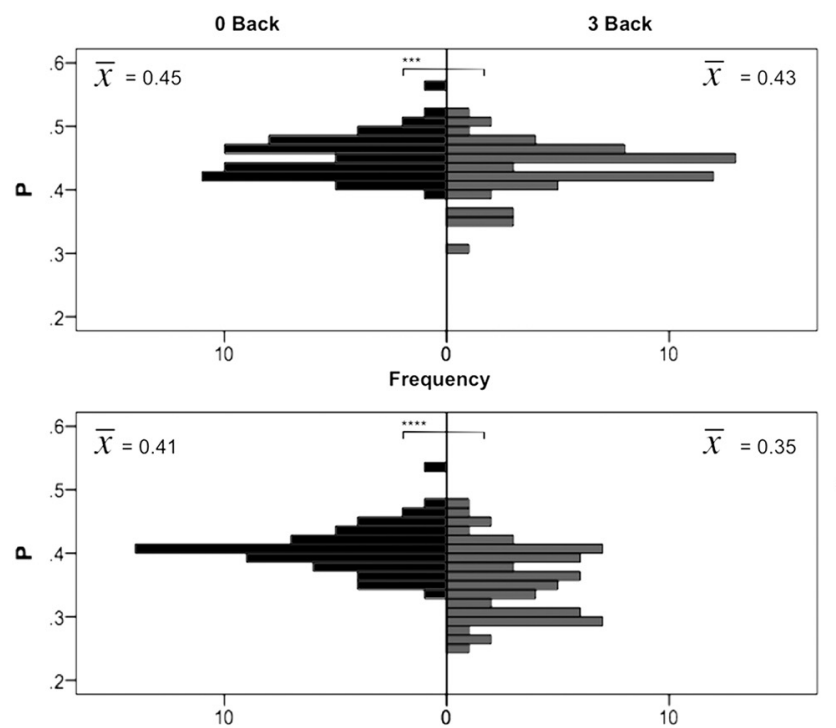

\section{B Nodal Strength (S)}

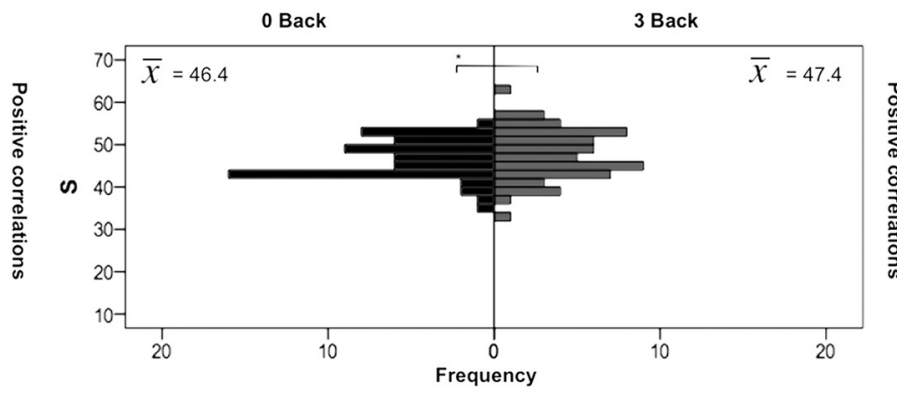

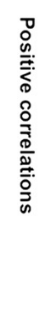

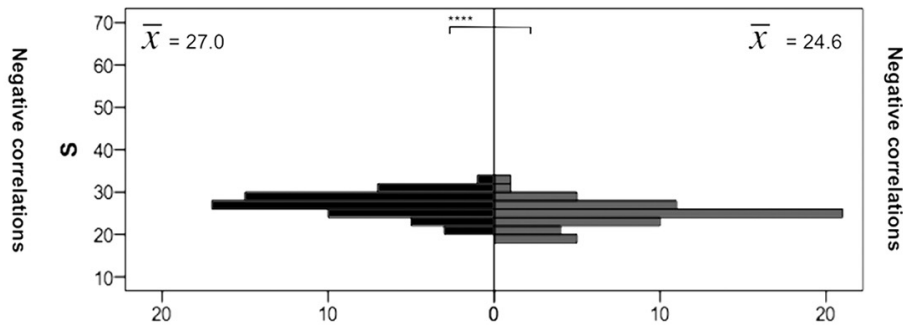

Figure 5. Nodal participation coefficient and strength measures for the positive and negative connections of DMN ROls at 0-Back and 3-Back conditions. $\boldsymbol{A}, \boldsymbol{B}$, While the nodal participation coefficient $(P)$ denotes the diversity of intermodular links $(\boldsymbol{A})$, the nodal strength $(S)$ represents the sum of positive and negative links made by each node $(\boldsymbol{B})$. The bars represent the histogram of frequency for given $P$ and $S$ values. The calculations were performed over 10 iterations and the paired $t$ tests at the 0.05 level of significance were controlled for multiple comparisons using Bonferroni's correction.

Together with our results, these findings also provide support for a relationship between changes in modularity and performance. Hence, the ability to transiently switch between a crystallized modular architecture to that of a highly integrated global workspace (Baars, 2002) with long-range connections may be related to human cognitive performance and conscious processing, such as in a working-memory task (Kitzbichler et al., 2011). The DMN with its observed flexible nodes across increasing cognitive loads may be facilitating such dynamic changes in global brain topography. As a caveat we need to mention that our study used a block design with low temporal resolution. To provide more conclusive evidence for the mechanism by which DMN nodes interact with other LSNs, future research will need to employ paradigms that occupy finer time scales. We also considered the possibility that the age range of the volunteers in this study may have weakened the overall impact of our findings. To this end, we included age as a confounding variable in our analyses where appropriate, and found that age had no effect on the associations we established between changes in modularity and reaction time to correct responses.

From a cognitive perspective, working memory constitutes a multicomponent system that retains and manipulates information for use in executive functions ranging from decision making to planning (Repovs and Baddeley, 2006). Thus, it represents an integral part of our everyday lives, allowing us to solve complex problems. Over the years, this hypothesis has been tested with various paradigms to assess the brain's response to "on-line" retention, updating, and manipulation of information with varying degrees of difficulty. Frontoparietal areas have been widely shown to activate in response to $n$-back tasks (Owen et al., 2005); however, growing evidence also highlights the DMNs' contribution to working memory.

Spreng and colleagues, for example, showed enhanced task performance when the task required access to long-term autobiographical memory stores supported by the DMN (Spreng et al., 2014). Using a novel famous-faces version of the $n$-back task, they reported greater DMN activity while participants matched famous as opposed to anonymous faces and concluded that the DMN's contribution may be restricted to accessing internal mental representations to facilitate congruent task goals. Expanding this hypothesis, in a perceptual version of the $n$-back, Konishi and colleagues showed greater activity in DMN, as well as in salience and frontoparietal networks, during 1-Back in comparison with 0 -Back conditions (Konishi et al., 2015). These results reinforced the assertion that regardless of autobiographical memory content, access to memory stores, as opposed to the processing of current perceptual input, was sufficient enough to drive DMN involvement (Smallwood, 2013). In light of these findings, the observed increase in volatility of the DMN regions and their interactions with other LSNs (e.g., salience and frontoparietal) during 1-Back as opposed to the 0-Back condition in our study (Fig. 3) might represent the $\mathrm{DMN}^{\prime}$ s transient retrieval of memory and integration of information for an expanded global workspace. Overall, this evidence suggests that, especially during paradigms that involve memory-based processing, the DMN may actively contribute to human cognition, a role that has not yet been fully defined.

In the context of segregation and integration in the brain, Baars developed the global workspace model related to conscious processing, in which the integration of information provides the necessary associations for reasoning, decision making, and planning (Baars, 2002). The interactions between the default mode, dorsal attention, and frontoparietal networks are hypothesized to engage with such dynamic and integrative processing in which the DMN is thought to provide internal information for global amplification facilitated by the frontoparietal network (Dehaene and Changeux, 2011; Smallwood et al., 2012). Along similar lines, the posterior cingulate has been discussed as an area that facilitates integration across multiple networks (Leech et al., 2012; Braga et al., 2013). Thus, with its extensive structural and functional connections, the DMN may constitute an important global workspace hub, providing associative information (Bar, 2007) 
for scrutiny and manipulation by the co-operating frontoparietal network. Such a framework would not only offer an explanation for the involvement of the DMN in a range of self-referential and memory-based tasks (Andrews-Hanna et al., 2014), but would also allude to its central importance in wider brain processing (Vatansever et al., 2015) that extends to social cognition and creativity (Wiggins and Bhattacharya, 2014).

A comparable concept was introduced by Baddeley (Baddeley, 2000), who argued for the existence of an episodic buffer, which integrates information from the visuospatial sketchpad, the phonological loop, and long-term memory stores for use by the central executive. Although there is no consensus on the neural correlates of the episodic buffer, the DMN's high structural and functional connectivity, its involvement in a wide variety of cognitive paradigms, and the potential contribution to the global integration of information, make the DMN a likely candidate for this role. Nevertheless, further research that directly investigates these hypotheses will be required to establish whether the DMN constitutes the neural underpinning of the theoretical global integrator and/or episodic buffer.

In conclusion, the results of our study demonstrate increasing interactions between various LSNs, including the DMN, with increasing cognitive effort during a working-memory task. In contrast to the historically held view on the irrelevance of DMN to goal-directed, attention-demanding paradigms, we propose that the DMN actively contributes to task performance, possibly through global integration of information, which might also explain its recently reported involvement in a diverse range of tasks. However, the precise cognitive mechanism that facilitates these processes remains a central question for future research.

\section{References}

Achard S, Salvador R, Whitcher B, Suckling J, Bullmore E (2006) A resilient, low-frequency, small-world human brain functional network with highly connected association cortical hubs. J Neurosci 26:63-72. CrossRef Medline

Andrews-Hanna JR (2012) The brain's default network and its adaptive role in internal mentation. Neuroscientist 18:251-270. CrossRef Medline

Andrews-Hanna JR, Smallwood J, Spreng RN (2014) The default network and self-generated thought: component processes, dynamic control, and clinical relevance. Ann N Y Acad Sci 1316:29-52. CrossRef Medline

Baars BJ (2002) The conscious access hypothesis: origins and recent evidence. Trends Cogn Sci 6:47-52. CrossRef Medline

Baddeley A (2000) The episodic buffer: a new component of working memory? Trends Cogn Sci 4:417-423. CrossRef Medline

Bar M (2007) The proactive brain: using analogies and associations to generate predictions. Trends Cogn Sci 11:280-289. CrossRef Medline

Bassett DS, Meyer-Lindenberg A, Achard S, Duke T, Bullmore E (2006) Adaptive reconfiguration of fractal small-world human brain functional networks. Proc Natl Acad Sci U S A 103:19518-19523. CrossRef Medline

Bassett DS, Wymbs NF, Porter MA, Mucha PJ, Carlson JM, Grafton ST (2011) Dynamic reconfiguration of human brain networks during learning. Proc Natl Acad Sci U S A 108:7641-7646. CrossRef Medline

Behzadi Y, Restom K, Liau J, Liu TT (2007) A component based noise correction method (CompCor) for BOLD and perfusion based fMRI. Neuroimage 37:90-101. CrossRef Medline

Blondel VD, Guillaume JL, Lambiotte R, Lefebvre E (2008) Fast unfolding of communities in large networks. J Stat Mech 10:P10008

Braga RM, Sharp DJ, Leeson C, Wise RJ, Leech R (2013) Echoes of the brain within default mode, association, and heteromodal cortices. J Neurosci 33:14031-14039. CrossRef Medline

Buckner RL, Andrews-Hanna JR, Schacter DL (2008) The brain's default network: anatomy, function, and relevance to disease. Ann N Y Acad Sci 1124:1-38. CrossRef Medline

Buckner RL, Sepulcre J, Talukdar T, Krienen FM, Liu H, Hedden T, AndrewsHanna JR, Sperling RA, Johnson KA (2009) Cortical hubs revealed by intrinsic functional connectivity: mapping, assessment of stability, and relation to Alzheimer's disease. J Neurosci 29:1860-1873. CrossRef Medline

Bullmore E, Sporns O (2009) Complex brain networks: graph theoretical analysis of structural and functional systems. Nat Rev Neurosci 10:186198. CrossRef Medline

Bullmore E, Sporns O (2012) The economy of brain network organization. Nat Rev Neurosci 13:336-349. CrossRef Medline

Chai XJ, Castañón AN, Ongür D, Whitfield-Gabrieli S (2012) Anticorrelations in resting state networks without global signal regression. Neuroimage 59:1420-1428. CrossRef Medline

Cohen AL, Fair DA, Dosenbach NU, Miezin FM, Dierker D, Van Essen DC, Schlaggar BL, Petersen SE (2008) Defining functional areas in individual human brains using resting functional connectivity MRI. Neuroimage 41:45-57. CrossRef Medline

Cole MW, Reynolds JR, Power JD, Repovs G, Anticevic A, Braver TS (2013) Multi-task connectivity reveals flexible hubs for adaptive task control. Nat Neurosci 16:1348-1355. CrossRef Medline

Damoiseaux JS, Rombouts SA, Barkhof F, Scheltens P, Stam CJ, Smith SM, Beckmann CF (2006) Consistent resting-state networks across healthy subjects. Proc Natl Acad Sci U S A 103:13848-13853. CrossRef Medline

Damoiseaux JS, Beckmann CF, Arigita EJ, Barkhof F, Scheltens P, Stam CJ, Smith SM, Rombouts SA (2008) Reduced resting-state brain activity in the "default network" in normal aging. Cereb Cortex 18:1856-1864. CrossRef Medline

Dehaene S, Changeux JP (2011) Experimental and theoretical approaches to conscious processing. Neuron 70:200-227. CrossRef Medline

De Luca M, Beckmann CF, De Stefano N, Matthews PM, Smith SM (2006) fMRI resting state networks define distinct modes of long-distance interactions in the human brain. Neuroimage 29:1359-1367. CrossRef Medline

de Pasquale F, Della Penna S, Snyder AZ, Marzetti L, Pizzella V, Romani GL, Corbetta M (2012) A cortical core for dynamic integration of functional networks in the resting human brain. Neuron 74:753-764. CrossRef Medline

de Pasquale F, Sabatini U, Della Penna S, Sestieri C, Caravasso CF, Formisano R, Péran P (2013) The connectivity of functional cores reveals different degrees of segregation and integration in the brain at rest. Neuroimage 69:51-61. CrossRef Medline

Dosenbach NU, Fair DA, Miezin FM, Cohen AL, Wenger KK, Dosenbach RA, Fox MD, Snyder AZ, Vincent JL, Raichle ME, Schlaggar BL, Petersen SE (2007) Distinct brain networks for adaptive and stable task control in humans. Proc Natl Acad Sci U S A 104:11073-11078. CrossRef Medline

Fair DA, Schlaggar BL, Cohen AL, Miezin FM, Dosenbach NU, Wenger KK, Fox MD, Snyder AZ, Raichle ME, Petersen SE (2007) A method for using blocked and event-related fMRI data to study "resting state" functional connectivity. Neuroimage 35:396-405. CrossRef Medline

Fox MD, Snyder AZ, Vincent JL, Corbetta M, Van Essen DC, Raichle ME (2005) The human brain is intrinsically organized into dynamic, anticorrelated functional networks. Proc Natl Acad Sci U S A 102:9673-9678. CrossRef Medline

Fox MD, Zhang D, Snyder AZ, Raichle ME (2009) The global signal and observed anticorrelated resting state brain networks. J Neurophysiol 101: 3270-3283. CrossRef Medline

Garrity AG, Pearlson GD, McKiernan K, Lloyd D, Kiehl KA, Calhoun VD (2007) Aberrant "default mode" functional connectivity in schizophrenia. Am J Psychiatry 164:450-457. CrossRef Medline

Green DM, Swets JA (1974) Signal detection theory and psychophysics. Huntington, NY: R. E. Krieger.

Guimerà R, Amaral LA (2005) Cartography of complex networks: modules and universal roles. J Stat Mech 2005:nihpa35573. Medline

Hagmann P, Cammoun L, Gigandet X, Meuli R, Honey CJ, Wedeen VJ, Sporns O (2008) Mapping the structural core of human cerebral cortex. PLoS Biol 6:e159-e159. CrossRef Medline

Hampson M, Driesen NR, Skudlarski P, Gore JC, Constable RT (2006) Brain connectivity related to working memory performance. J Neurosci 26:13338-13343. CrossRef Medline

Hoaglin DC, Iglewicz B, Tukey JW (1986) Performance of some resistant rules for outlier labeling. J Am Stat Assoc 81:991-999. CrossRef

Kelly AM, Uddin LQ, Biswal BB, Castellanos FX, Milham MP (2008) Competition between functional brain networks mediates behavioral variability. Neuroimage 39:527-537. CrossRef Medline

Kitzbichler MG, Henson RN, Smith ML, Nathan PJ, Bullmore ET (2011) 
Cognitive effort drives workspace configuration of human brain functional networks. J Neurosci 31:8259-8270. CrossRef Medline

Konishi M, McLaren DG, Engen H, Smallwood J (2015) Shaped by the past: the default mode network supports cognition that is independent of immediate perceptual input. PLoS One 10:e0132209. CrossRef Medline

Kruschwitz JD, List D, Waller L, Rubinov M, Walter H (2015) GraphVar: A user-friendly toolbox for comprehensive graph analyses of functional brain connectivity. J Neurosci Methods 245:107-115. CrossRef Medline

Leech R, Braga R, Sharp DJ (2012) Echoes of the brain within the posterior cingulate cortex. J Neurosci 32:215-222. CrossRef Medline

Li SC, Sikström S (2002) Integrative neurocomputational perspectives on cognitive aging, neuromodulation, and representation. Neurosci Biobehav Rev 26:795-808. CrossRef Medline

Mazoyer B, Zago L, Mellet E, Bricogne S, Etard O, Houdé O, Crivello F, Joliot M, Petit L, Tzourio-Mazoyer N (2001) Cortical networks for working memory and executive functions sustain the conscious resting state in man. Brain Res Bull 54:287-298. CrossRef Medline

Meunier D, Achard S, Morcom A, Bullmore E (2009a) Age-related changes in modular organization of human brain functional networks. Neuroimage 44:715-723. CrossRef Medline

Meunier D, Lambiotte R, Fornito A, Ersche KD, Bullmore ET (2009b) Hierarchical modularity in human brain functional networks. Front Neuroinform 3:37. CrossRef Medline

Meunier D, Stamatakis EA, Tyler LK (2014) Age-related functional reorganization, structural changes, and preserved cognition. Neurobiol Aging 35:42-54. CrossRef Medline

Murphy K, Birn RM, Handwerker DA, Jones TB, Bandettini PA (2009) The impact of global signal regression on resting state correlations: are anticorrelated networks introduced? Neuroimage 44:893-905. CrossRef Medline

Newman ME (2006) Modularity and community structure in networks. Proc Natl Acad Sci U S A 103:8577-8582. CrossRef Medline

Nooy Wd, Mrvar A, Batagelj V (2011) Exploratory social network analysis with Pajek, revised and expanded 2nd edition. New York: Cambridge UP.

Owen AM, McMillan KM, Laird AR, Bullmore E (2005) N-back working memory paradigm: a meta-analysis of normative functional neuroimaging studies. Hum Brain Mapp 25:46-59. CrossRef

Power JD, Cohen AL, Nelson SM, Wig GS, Barnes KA, Church JA, Vogel AC, Laumann TO, Miezin FM, Schlaggar BL, Petersen SE (2011) Functional network organization of the human brain. Neuron 72:665-678. CrossRef

Raichle ME, MacLeod AM, Snyder AZ, Powers WJ, Gusnard DA, Shulman GL (2001) A default mode of brain function. Proc Natl Acad Sci U S A 98:676-682. CrossRef

Repovs G, Baddeley A (2006) The multi-component model of working memory: explorations in experimental cognitive psychology. Neuroscience 139:5-21. CrossRef

Rosvall M, Bergstrom CT (2010) Mapping change in large networks. PLoS One 5:e8694. CrossRef

Rosvall M, Axelsson D, Bergstrom CT (2009) The map equation. Eur Phys J Spec Top 178:13-23. CrossRef

Rubinov M, Sporns O (2010) Complex network measures of brain connectivity: uses and interpretations. Neuroimage 52:1059-1069. CrossRef Medline

Rubinov M, Sporns O (2011) Weight-conserving characterization of complex functional brain networks. Neuroimage 56:2068-2079. CrossRef Medline

Sala-Llonch R, Peña-Gómez C, Arenaza-Urquijo EM, Vidal-Piñeiro D, Bargalló N, Junqué C, Bartrés-Faz D (2012) Brain connectivity during resting state and subsequent working memory task predicts behavioural performance. Cortex 48:1187-1196. CrossRef Medline

Sharp DJ, Beckmann CF, Greenwood R, Kinnunen KM, Bonnelle V, De Boissezon X, Powell JH, Counsell SJ, Patel MC, Leech R (2011) Default mode network functional and structural connectivity after traumatic brain injury. Brain 134:2233-2247. CrossRef Medline

Shulman GL, Fiez JA, Corbetta M, Buckner RL, Miezin FM, Raichle ME,
Petersen SE (1997) Common blood flow changes across visual tasks: II. Decreases in cerebral cortex. J Cogn Neurosci 9:648-663. CrossRef Medline

Smallwood J (2013) Distinguishing how from why the mind wanders: a process-occurrence framework for self-generated mental activity. Psychol Bull 139:519-535. CrossRef Medline

Smallwood J, Brown K, Baird B, Schooler JW (2012) Cooperation between the default mode network and the frontal-parietal network in the production of an internal train of thought. Brain Res 1428:60-70. CrossRef Medline

Spreng RN (2012) The fallacy of a "task-negative" network. Front Psychol 3:145. CrossRef Medline

Spreng RN, Grady CL (2010) Patterns of brain activity supporting autobiographical memory, prospection, and theory of mind, and their relationship to the default mode network. J Cogn Neurosci 22:1112-1123. CrossRef Medline

Spreng RN, Stevens WD, Chamberlain JP, Gilmore AW, Schacter DL (2010) Default network activity, coupled with the frontoparietal control network, supports goal-directed cognition. Neuroimage 53:303-317. CrossRef Medline

Spreng RN, Sepulcre J, Turner GR, Stevens WD, Schacter DL (2013) Intrinsic architecture underlying the relations among the default, dorsal attention, and frontoparietal control networks of the human brain. J Cogn Neurosci 25:74-86. CrossRef Medline

Spreng RN, DuPre E, Selarka D, Garcia J, Gojkovic S, Mildner J, Luh WM, Turner GR (2014) Goal-congruent default network activity facilitates cognitive control. J Neurosci 34:14108-14114. CrossRef Medline

Stamatakis EA, Adapa RM, Absalom AR, Menon DK (2010) Changes in resting neural connectivity during propofol sedation. PLoS One 5:e14224-e14224. CrossRef Medline

Stamatakis EA, Shafto MA, Williams G, Tam P, Tyler LK (2011) White matter changes and word finding failures with increasing age. PLoS One 6:e14496. CrossRef Medline

Stanley ML, Dagenbach D, Lyday RG, Burdette JH, Laurienti PJ (2014) Changes in global and regional modularity associated with increasing working memory load. Front Hum Neurosci 8:954. CrossRef Medline

Stevens AA, Tappon SC, Garg A, Fair DA (2012) Functional brain network modularity captures inter- and intra-individual variation in working memory capacity. PLoS One 7:e30468. CrossRef Medline

van den Heuvel MP, Sporns O (2011) Rich-club organization of the human connectome. J Neurosci 31:15775-15786. CrossRef Medline

van den Heuvel MP, Sporns O (2013) Network hubs in the human brain. Trends Cogn Sci 17:683-696. CrossRef Medline

van den Heuvel MP, Stam CJ, Kahn RS, Hulshoff Pol HE (2009) Efficiency of functional brain networks and intellectual performance. J Neurosci 29:7619-7624. CrossRef Medline

Vatansever D, Menon DK, Manktelow AE, Sahakian BJ, Stamatakis EA (2015) Default mode network connectivity during task execution. Neuroimage 122:96-104. CrossRef Medline

Whitfield-Gabrieli S, Nieto-Castanon A (2012) Conn: a functional connectivity toolbox for correlated and anticorrelated brain networks. Brain Connectivity 2:125-141. CrossRef Medline

Whitfield-Gabrieli S, Thermenos HW, Milanovic S, Tsuang MT, Faraone SV, McCarley RW, Shenton ME, Green AI, Nieto-Castanon A, LaViolette P, Wojcik J, Gabrieli JD, Seidman LJ (2009) Hyperactivity and hyperconnectivity of the default network in schizophrenia and in first-degree relatives of persons with schizophrenia. Proc Natl Acad Sci U S A 106: 1279-1284. CrossRef Medline

Wig GS, Schlaggar BL, Petersen SE (2011) Concepts and principles in the analysis of brain networks. Ann N Y Acad Sci 1224:126-146. CrossRef Medline

Wiggins GA, Bhattacharya J (2014) Mind the gap: an attempt to bridge computational and neuroscientific approaches to study creativity. Front Hum Neurosci 8:540. CrossRef Medline 